\title{
Lumen
}

Selected Proceedings from the Canadian Society for Eighteenth-Century Studies

\section{Imitation des Anciens et invention dans les Éléments de littérature de Marmontel}

\section{Youmna Charara}

Volume 26, 2007

Imitation et invention au siècle des Lumières

Imitation and Invention in the Eighteenth Century

URI : https://id.erudit.org/iderudit/1012060ar

DOI : https://doi.org/10.7202/1012060ar

Aller au sommaire du numéro

Éditeur(s)

Canadian Society for Eighteenth-Century Studies / Société canadienne d'étude du dix-huitième siècle

ISSN

1209-3696 (imprimé)

1927-8284 (numérique)

Découvrir la revue

Citer cet article

Charara, Y. (2007). Imitation des Anciens et invention dans les Éléments de littérature de Marmontel. Lumen, 26, 49-62. https://doi.org/10.7202/1012060ar

Copyright (c) Canadian Society for Eighteenth-Century Studies / Sociéte canadienne d'étude du dix-huitième siècle, 2007
Ce document est protégé par la loi sur le droit d'auteur. L'utilisation des services d'Érudit (y compris la reproduction) est assujettie à sa politique d'utilisation que vous pouvez consulter en ligne.

https://apropos.erudit.org/fr/usagers/politique-dutilisation/ 


\section{Imitation des Anciens et invention dans les Éléments de littérature de Marmontel}

Les rapports entre Anciens et Modernes révèlent leur caractère problématique, dans les Éléments de littérature de Marmontel, à travers trois types de discours. Un discours normatif, adressé aux auteurs, délimitant la part réservée à l'imitation, indique les moyens d'un perfectionnement de l'héritage littéraire et les conditions d'une rupture avec les modèles antiques. Les Éléments sont aussi le lieu d'un discours historique qui analyse les différences entre littérature moderne et littérature gréco-latine. La description d'évolutions formelles et thématiques s'accompagne d'explications sociologiques, religieuses et politiques qui rendent compte des spécificités de chaque époque ; elle permet de discerner les composantes génériques, qui traversent le temps, et les composantes idiomatiques, indissociables d'un contexte culturel singulier, qui restreignent le champ de l'imitation. Le discours philosophique, ou le discours de philosophie de l'histoire, refuse de se prêter au «rituel agonistique ${ }^{1} \gg$ de la hiérarchisation des périodes littéraires : jugeant par trop simplificatrices les généralisations qui exacerbent la Querelle des Anciens et des Modernes, il contribue à la liquidation de cette polémique au moyen, notamment, d'une fragmentation des énoncés évaluatifs, qui s'appliquent à un texte, ou à une partie de texte, plutôt qu'à une culture prise dans son intégralité. L'accent est mis, dans cette perspective, sur l'hétérogénéité de l'Iliade ou de l'Énéide, admirables ou manqués selon les pages, pour des raisons dont le critique littéraire doit rendre toute la complexité. La philosophie de l'histoire s'efface devant l'analyse d'œuvres singulières.

C'est dans le cadre d'une confrontation entre Anciens et Modernes que Marmontel s'interroge véritablement sur la possibilité de l'invention

1 L'expression est de Marc Fumaroli, La Querelle des Anciens et des Modernes, Paris, Gallimard, coll. «Folio classique», 2001, p. 219-220. 
littéraire. Si Roland Mortier a jugé décevante l'enquête sur la notion d'originalité dans les Éléments de littérature, il faut en chercher la raison, sans doute, dans des choix de lecture qui privilégient le discours de théorie esthétique de Marmontel. Les valeurs d'authenticité, de singularité, de fidélité à l'originel seraient faiblement représentées dans les Éléments, selon R. Mortier, alors qu'elles s'épanouissent dans les œuvres de Rousseau et de Diderot ; soumise au règne de l'imitation et aux idéaux de l'âge classique, la pensée conformiste de Marmontel accuserait un temps de retard. De fait, $\mathrm{l}^{\prime}$ «Essai sur le goût» qui sert d'introduction à l'ouvrage, ainsi que les articles «Beau», «Enthousiasme» ou "Génie», développent, abstraction faite de quelques «saillies», des théories esthétiques conventionnelles, qui amènent $R$. Mortier à conclure légitimement : «La notion d'originalité s'estompe, dans les Éléments, au profit de celle de règle»; "Marmontel n'a fait des concessions à un vague primitivisme et à une esthétique de la sincérité que pour mieux les reléguer dans un passé révolu ${ }^{2}$ ». C'est peut-être en dehors de la théorie que se prépare une culture littéraire affranchie des dogmes classiques; la réflexion sur les exemples et les pratiques concrètes trahit quelquefois, à l'insu de l'auteur, une mutation des valeurs esthétiques. Nous faisons l'hypothèse selon laquelle Marmontel poéticien et historien de la littérature participe à sa manière à la promotion de l'idéal d'originalité au XVIII ${ }^{\mathrm{e}}$ siècle ${ }^{3}$.

Marmontel se situe dans le cadre d'une culture rhétorique, sa référence constante à Cicéron et sa conception du texte comme relais permettant la création d'œuvres nouvelles en constituent les signes les

2 Roland Mortier, L'Originalité. Une nouvelle catégorie esthétique au siècle des Lumières, Genève, Droz, 1982, p. 184. Sur les théories littéraires et esthétiques de Marmontel voir aussi Annie Becq, «Les idées esthétiques de Marmontel», dans Jean Ehrard (études réunies et présentées par), De l'Encyclopédie à la Contre-Révolution. Jean-François Marmontel (1723-1799), Clermont-Ferrand, G. de Bussac, 147-174. A. Becq parle d'un "dosage de vues hardies et traditionnelles», et de "souplesse éclectique», le beau étant caractérisé dans les Éléments tantôt par une évidence rationnelle, tantôt par l'irrégularité, voire l'absurdité ; la faculté du goût est quelquefois identifiée à la raison intellectuelle, et parfois à un instinct ou un sentiment.

3 Pour une étude d'ensemble des Éléments de littérature, voir Michael Cardy, The Literary Doctrines of Jean-François Marmontel, Oxford, The Voltaire Foundation, coll. "Studies on Voltaire and the Eighteenth Century ", 1982. M. Cardy analyse l'évolution de la pensée de Marmontel, sous l'influence du contexte intellectuel et des débats de l'époque ; il attire l'attention sur le fait que les Éléments de littérature, publiés en 1787, rassemblent des articles de Marmontel (d'abord parus dans l'Encyclopédie, le Supplément et l'Encyclopédie méthodique) dont la publication s'étale sur près de quarante ans. 
plus manifestes. Cette tradition valorise certains types d'invention, et en interdit d'autres. Des idées neuves se dégagent lentement de la pensée rhétorique, Marmontel procédant par des reformulations de la doctrine de l'imitation qui s'accompagnent de décalages imperceptibles, de déplacements d'accent - plutôt que par «fulgurances» théoriques. L'œuvre fictionnelle de Marmontel peut apporter un éclairage précieux sur le rapport avec les modèles antiques dont l'auteur moderne fait l'expérience dans son travail de création ; sans présupposer une coïncidence absolue entre les textes critiques et les conceptions esthétiques qui sous-tendent les romans de Marmontel, il paraît légitime de rechercher les points de rencontre qui valident une interprétation des Éléments de littérature.

L'article «Imitation» manifeste une foi vibrante dans les vertus de cette pratique d'écriture : Molière, Racine, La Fontaine ne sont après tout que des imitateurs de génie. Marmontel souligne la nécessité de vivifier l'imitation, dégradée par «nos anciens régents», qui l'ont réduite à un exercice scolaire exsangue, et par les écrivains sans talent, qui se bornent au décalque servile de l'original. «Troupeau d'esclaves», dit Marmontel à propos de la masse des imitateurs. L'imitation est une épreuve héroïque, qui implique l'émulation, et ne laisse pas d'autre alternative que celle de l'échec humiliant ou de la gloire.

La «méthode d'imitation» proposée, qui a la caution de Cicéron, exige un travail de sélection qui recueille le meilleur de l'œuvre imitée et répudie les parties faibles du modèle ${ }^{4}$. Elle doit être complétée par la théorie du "modèle intellectuel», attribuée également à Cicéron : à partir des beautés existantes nécessairement imparfaites (réussites artistiques ou merveilles de la nature), l'artiste se forme un «type» transcendant, qu'il s'efforce ensuite de traduire dans son œuvre («Critique», 315-318) ${ }^{5}$. L'artiste imite en définitive un tout idéal, qui n'existe que dans la pensée, composé d'une multitude de beautés éparses ${ }^{6}$. Ainsi peut s'expliquer le progrès accompli par l'imitateur par rapport au

4 Marmontel évoque également dans l'article «Imitation» une deuxième méthode d'imitation, attribuée à Longin, mais il s'agit d'un hapax dans les Éléments de littérature ; l'inspiration cicéronienne domine largement dans cet ouvrage.

5 Les citations proviennent des Éléments de littérature de Marmontel, édition présentée, établie et annotée par Sophie Le Ménahèze, Paris, Desjonquères, 2005 (cette édition reprend celle de 1787). Nous indiquons entre parenthèses le titre de l'article et le numéro de la page.

6 Voir aussi l'article «Invention poétique» (674) : «Celui qui compose un tout idéal, intéressant et nouveau, d'un assemblage de choses connues, ou qui donne à un 
texte original. Une poétique et une rhétorique de l'imitation viennent corroborer la loi qui s'impose à l'imitateur «d'embellir ses modèles»; toutes les formes de réécriture évoquées impliquent une amélioration du texte-source: "Faire passer dans un nouvel ouvrage des beautés étrangères, anciennes ou modernes, et dont on enrichit sa langue ; [...] dans sa langue même, recueillir d'un ouvrage obscur et oublié des pensées heureuses, mais indignement mises en œuvre par l'inventeur, et les placer, les assortir, les exprimer comme elles devaient l'être ${ }^{7} »$; le traducteur rehausse les qualités de l'œuvre originale, sans s'astreindre à l'exactitude littérale ${ }^{8}$.

L'invention, dans ce contexte, a pour signe distinctif non pas l'originalité mais la perfection, dans un rapport de rivalité avec des modèles littéraires particuliers, et d'adéquation plus ou moins achevée au «modèle intellectuel».

Plusieurs types d'invention sont analysés dans les Éléments. Si on excepte le cas hypothétique de l'invention qui procède exclusivement de l'imitation de la nature ${ }^{9}$, il faut distinguer une première forme d'invention caractérisée par la continuité avec les textes-sources, dont Virgile pourrait être la figure emblématique, et une deuxième forme marquée par la rupture avec les modèles, ou du moins par un bouleversement de l'héritage littéraire, dont l'œuvre de Corneille est le prototype le plus représentatif ; une invention d'un troisième genre, liée à une promotion de l'énonciateur, pour laquelle Marmontel ne donne que des exemples, s'impose dans certains articles, sans posséder le même statut canonique que les précédentes.

"Virgile a peint le plus beau tableau qui nous reste de l'Antiquité»-le contraste du trouble de Didon et de la quiétude de la nuit - $\mathrm{d}^{\prime}$ après une esquisse d'Apollonius de Rhodes qu'il a enrichie en lui conférant l'unité et la noblesse d'expression qui lui faisaient défaut («Imitation», 658659) ; Racine a surpassé Euripide ; Molière est très supérieur à Plaute,

tout existant une vie, une grâce, une beauté nouvelle ; celui-là, dis-je, est poète, ou Corneille et Homère ne le sont pas».

7 «Imitation», p. 657 ; nous soulignons.

8 Marmontel traducteur de la Pharsale s'impose lui-même cette règle; il écrit dans la préface à sa traduction : «Ce poème avait donc besoin d'être traduit non pas servilement, mais avec choix, avec intelligence» (Marmontel, CEuvres complètes, Genève, Slatkine Reprints, 1968, t. 6, p. 526).

9 «Nous connaissons quelques hommes extraordinaires, tels qu'Homère et Eschyle, qui semblent n'avoir eu pour modèle que la nature et pour guide que leur instinct ; mais est-il bien sûr qu'avant Homère l'art de la poésie n'eût pas été cultivé, raisonné, soumis à des lois ?» («Règles», 990). 
etc. Le premier type d'invention se caractérise donc par le respect d'un cadre générique; le second, par la transformation des structures du genre hérité. Corneille a opéré une "psychologisation» de la tragédie, qui partage en deux l'histoire de ce genre: au «système ancien», où le héros est esclave de la destinée, s'oppose depuis Corneille «le système moderne», où le personnage est esclave de ses passions ; «dès lors le ressort de l'action tragique a été le cœur de l'homme» («Tragédie», 1087-1100). Le théâtre cornélien effectue ce que Gérard Genette appelle un travail de motivation qui «fonctionne comme intériorisation d'une cause externe ${ }^{10}{ }^{\prime}$. L'inventeur abandonne les composantes idiomatiques des modèles antiques - les «beautés locales»- étroitement associées aux croyances religieuses des Grecs; il retient les composantes essentielles du genre. L'invention n'est possible, dans ce cas, qu'au prix d'une transgression des règles, déduites de l'exemple des Anciens; Aristote exigeant que le héros fût l'artisan aveugle de son malheur, Corneille aurait sacrifié les sujets du plus grand nombre de ses pièces, s'il n'était, fort heureusement, un génie libre («Règles», 993). De toute évidence, $c^{\prime}$ est le second type d'invention qui a les faveurs de Marmontel ; les auteurs de l'Antiquité, dit-il, «n'ont acquis le droit de commander que parce qu'ils n'ont pas obéi» («Critique», 326); et "comment ressembler à celui qui ose, si on n'ose pas comme lui ?» («Imitation», 656). Il arrive ainsi à Marmontel de regretter le poids excessif de la culture littéraire ; l'autorité des modèles antiques ne permettrait pas d'éduquer le goût et de perfectionner le «modèle intellectuel», mais constituerait une entrave, en imposant aux auteurs des contraintes désuètes: «Presque tous les grands génies, depuis Homère jusqu'à Lucrèce, depuis Lucrèce jusqu'à Corneille, semblent avoir choisi, pour s'élever, les temps où l'ignorance leur laissait une libre carrière ${ }^{11}{ }^{\prime}$. La valorisation de la rupture introduite par Corneille, que l'exemple d'un Shakespeare aurait conduit à relativiser, et la dépréciation de l'héritage historique, jugé encombrant, participent de l'élaboration d'une nouvelle culture esthétique. Mais Marmontel défend la modernité dans des termes anciens ${ }^{12}$;

10 Gérard Genette, Palimpsestes. La littérature au second degré, Paris, Éditions du Seuil, coll. «Poétique », 1982, p. 375.

11 "Critique», 326 ; nous soulignons.

12 Jacques Wagner a souligné le caractère conciliateur de la pensée marmontélienne ; voir notamment «Marmontel ou l'horreur du conflit», dans J. Wagner (études réunies et présentées par), Marmontel, une rhétorique de l'apaisement, Louvain, Peeters, 2003, 5-16 ; et «Les deux personnalités», dans J. Wagner (dir.), Marmontel, un intellectuel exemplaire au siècle des Lumières, Tulle (France), Mille Sources, 2003, 83-99. 
le passage est presque insensible entre l'esthétique de l'écart, propre à l'imitation rhétorique, et l'idée d'un génie créateur en rupture avec le passé.

Les Éléments de littérature appellent à de nouvelles inventions, également fondées sur des transformations génériques, justifiées par la théorie de l'imitation sélective et la critique partielle des modèles antiques ; elles prennent le plus souvent la forme de l'hybridation générique, pour laquelle l'auteur manifeste une prédilection particulière. "Tout n'est pas beau chez les Anciens» («Règles», 993); les épopées en particulier manquent de tension dramatique, ou de «suspense» : «L'intrigue a été jusqu'ici la partie la plus négligée du poème épique, tandis que dans la tragédie elle s'est perfectionnée de plus en plus. On a osé se détacher de Sophocle et d'Euripide ; mais on a craint d'abandonner les traces d'Homère : Virgile l'a imité, et l'on a imité Virgile» («Épopée», 515). Bien qu'il se réclame d'Aristote, qui définit l'épopée comme une tragédie en récit, et de l'exemple du livre IV de l'Énéide, «ce mélange d'épique et de dramatique», pour recommander une forte concentration de l'intrigue, Marmontel ne programme pas un retour aux origines de l'épopée, dans un souci de fidélité au passé, mais une hybridation générique qui croise l'épopée antique et la tragédie moderne. Et comme c'est le roman qui offre les exemples d'intérêt dramatique les plus accomplis, Marmontel en arrive à préconiser la création d'œuvres hybrides alliant les structures de l'épopée antique et du roman moderne.

Je ne prétends pas comparer en tous points le mérite d'un beau roman avec celui d'un beau poème : mais qu'il me soit permis de demander pourquoi certains romans nous touchent, nous remuent, nous attachent $[\ldots]$; tandis que nous lisons d'un œil sec, je dis plus, tandis que nous lisons à peine sans une espèce de langueur les plus beaux poèmes épiques. C'est que dans ces romans le pathétique règne d'un bout à l'autre; au lieu que dans ces poèmes il n'occupe que des intervalles, et qu'il y est souvent négligé ${ }^{13}$.

Le Télémaque lui-même, chef-d'œuvre moderne, suscite le même ennui que l'épopée homérique ou virgilienne, faute d'intégrer les apports du roman pathétique ; le texte mixte dont Marmontel indique la possibilité suppose une expérimentation formelle plus audacieuse, non sans risque de déséquilibre et de fracture. La dramatisation et la psychologisation de l'épopée ne peuvent qu'entraîner le démantèlement d'un genre fondé sur la cohésion du héros et du monde, sur la convenance 
des actes à un idéal de grandeur. Toutefois, l'hybridation générique, pratique répandue au XVIII ${ }^{\mathrm{e}}$ siècle, ne pose pas problème à Marmontel. «Plus passionnés», écrit-il, les caractères d'Ulysse et de Télémaque

n'en seraient que plus touchants; et lorsque Télémaque s'arrache au plaisir, on aimerait encore mieux qu'il cédât aux mouvements de la nature qu'aux froids conseils de la sagesse. Si ce poème divin du côté de la morale laisse désirer quelque chose, c'est plus de chaleur et de pathétique ; et c'est aussi ce qui manque à l'Odyssée et à la plupart des poèmes connus ${ }^{14}$.

Il ne faudrait sans doute pas opposer de manière trop schématique la pureté générique dans la théorie et la littérature classiques à l'éclatement des genres à partir du XIX ${ }^{e}$ siècle ou, comme l'écrit Jean-Marie Schaeffer étudiant l'histoire des genres, "un passé pétrifié à un présent en mouvement ${ }^{15}$ ». L'abbé Batteux justifie l'opéra en le rapprochant de l'épopée : «C'est le divin de l'épopée mis en spectacle ${ }^{16}$ ». Le Bossu légitime son interprétation allégorique de l'épopée par une assimilation du poème épique et de l'apologue, genre illustré par les fables d'Ésope, situées à un degré très inférieur dans la hiérarchie des genres ${ }^{17}$. Le cloisonnement générique n'a jamais été respecté absolument. Empruntant à ces prédécesseurs, Marmontel poéticien se distingue toutefois par des propositions de rénovation des genres littéraires et d'invention de formes inédites. Il conjugue deux démarches, l'une historique, l'autre normative. L'étude diachronique des genres donne lieu à des comparaisons entre Anciens et Modernes qui ne laissent pas entièrement indemnes les «modèles» de l'Antiquité ; ce discours historique confère une assise à un discours prescriptif, qui explore de nouvelles combinaisons, ima-

14 Id.

15 Jean-Marie Schaeffer, «Les genres littéraires, d'hier à aujourd'hui», dans Marc Dambre et Monique Gosselin-Noat (dir.), L'éclatement des genres au XX' siècle, Paris, Presses de la Sorbonne Nouvelle, 2001, 11-20. J.-M. Schaeffer met en garde contre l'influence que le présent exerce quelquefois sur le passé : «C'est la thèse de la fin des genres qui institue le passé en monde générique clos» ; il juge difficile, néanmoins, de remettre en question cette clôture dans le cas de la littérature française «littérature dans laquelle la notion d'une norme générique correcte - fonctionnant presque à l'instar d'une norme grammaticale - a pendant longtemps été un thème central» (p. 15 et 13).

16 Charles Batteux, Les beaux-arts réduits à un même principe (1746), édition critique par Jean-Rémy Mantion, Paris, Aux Amateurs de livres, 1989, p. 211.

17 René Le Bossu, Traité du poème épique (1675), réimpr. de l'éd. de 1714 avec une introduction de Volker Kapp, Hamburg, Helmut Buske Verlag, 1981, p. 37 et s. 
gine des modèles génériques à venir, non encore réalisés, non conformes à certains égards aux textes anciens. Les Éléments de littérature diffèrent par là des «arts poétiques» classiques dont les règles sont entièrement abstraites des œuvres exemplaires du passé. Pour reprendre ses propres termes, Marmontel ne donne jamais «les faits pour la limite des possibles ${ }^{18} "$.

L'expérience de Marmontel romancier rejoint la réflexion du critique. Les Incas, ou la destruction de l'Empire du Pérou (1777), poème en prose qui multiplie les signes d'appartenance à l'épopée, constitue un exemple de croisement générique. "Quant à la forme de cet ouvrage, considéré comme une production littéraire, » écrit Marmontel dans la préface, « je ne sais, je l'avoue, comment le définir. Il y a trop de vérité pour un roman, et pas assez pour une histoire. Je n'ai certainement pas eu la prétention de faire un poème ${ }^{19}{ }^{\prime}$. Rỏman, histoire, épopée ? Il est possible également de considérer l'ouvrage comme un roman philosophique, ou de le classer parmi les «romans politiques», pour reprendre une catégorie forgée par Marmontel lui-même dans l'Essai sur les romans. Le ton modeste et l'embarras que provoque la tentative d'identification générique suggèrent que l'hybridation est une pratique spontanée, dépourvue de caution théorique. Les Incas et les Éléments de littérature s'inspirent de modalités d'écriture de l'époque, et sont en avance par rapport à la production de valeurs littéraires qui pourraient légitimer ces nouvelles formes d'invention. Étrangers à toute intention provocatrice de déstabilisation de l'héritage littéraire, ils s'inscrivent néanmoins dans la tendance à «l'accélération du rythme des transformations génériques» qui caractérise la littérature des $\mathrm{XIX}^{\mathrm{e}}$ et $\mathrm{X} \mathrm{X}^{\mathrm{e}}$ siècles ${ }^{20}$.

Les auteurs les plus admirés de Marmontel ont procédé dans leur imitation des modèles littéraires à des transformations énonciatives, qui affectent la relation avec le destinataire et la visée pragmatique de

18 Marmontel écrit dans une attaque contre Scaliger et Dacier : «Voilà comme une théorie exclusivement attachée à la pratique des Anciens donne les faits pour la limite des possibles, et veut réduire le génie à l'éternelle servitude d'une étroite imitation» ("Règles ", 106). La démarche de Marmontel tient à la fois de l'attitude prescriptive des théoriciens classiques, dans leurs classifications des genres, et des conceptions évolutionnistes des historiens de la littérature du XIX ${ }^{e}$ siècle, qui étudient la genèse et l'évolution des genres, en expliquant les modifications génériques par l'influence de la race, du climat, de la société et du génie individuel des créateurs. Sur les différentes théories génériques, voir Jean-Marie Schaeffer, Qu'estce qu'un genre littéraire?, Paris, Éditions du Seuil, coll. «Poétique», 1989.

19 Marmontel, Les Incas, Paris, Lacombe, 1777, préface, t. 1, p. 18.

20 Jean-Marie Schaeffer, «Les genres littéraires, d'hier à aujourd'hui», art. cit, p. 17. 
l'œuvre. Cette invention manque de visibilité dans les Éléments de littérature, parce qu'elle se révèle à travers des exemples, dans un "silence théorique» presque complet, mais elle réalise dans sa plénitude, aux yeux de Marmontel, la vocation de la littérature.

Fénelon et Voltaire sont les créateurs de la poésie épique en France ; "qu'ont fait les hommes de génie qui, dans l'épopée, ont voulu donner à la poésie française un plus heureux essor [que celui donné par SaintAmant, Desmarets, Chapelain] ?» («Poésie», 921). Fénelon «a ramené la poésie dans son berceau et aux pieds du tombeau d'Homère. Il a pris son sujet dans Homère lui-même» ; cet imitateur a innové par son style, par la relation pédagogique instaurée avec le destinataire princier, et par son courage politique, dans le contexte de la monarchie absolue :

au milieu de tous les trésors que nous avons vus étalés dans la Grèce sous les mains de la poésie, il en a pris en liberté, mais avec le discernement du goût le plus exquis, tout ce qui pouvait rendre aimable, intéressante et persuasive, la plus courageuse leçon qu'on ait jamais donnée aux enfants de nos rois ${ }^{21}$.

Attribuant à la littérature une mission morale et pédagogique, Marmontel n'est pas loin de sacraliser les œuvres susceptibles de communiquer l'amour de l'humanité et de défendre la justice. Le Télémaque est un "poème divin du côté de la morale». L'invention éthique associe l'excentricité de la position énonciative et l'universalité du message. Lucain, Fénelon, Voltaire ont régénéré la littérature en imposant leur voix, grâce à leur autorité morale d'écrivains extérieurs à la sphère du pouvoir. «Lucain est surtout recommandable par la hardiesse avec laquelle il a traité son sujet aux yeux des Romains, devenus esclaves, et dans la cour de leur tyran [...]. Ce génie audacieux avait senti qu'il était naturel à tous les hommes d'aimer la liberté ${ }^{22}{ }$. Inventeur qui s'écarte des leçons apprises, Lucain est aussi considéré comme une source nouvelle pour les imitateurs modernes, susceptible de régénérer l'écriture épique.

La Pharsale - dont Marmontel a donné une traduction en 1766 - se distingue par une inscription particulièrement adéquate de l'auteur dans un moment historique singulier; Lucain joue dans un contexte d'oppression le rôle admirable d'un écrivain républicain. L'insistance sur la situation d'énonciation s'oppose à la dévalorisation classique de ce qui rappelle le présent de l'écriture, souvent assimilé à «la mode», à «l'opinion», toutes deux passagères («Convenance», p. 306-307). La

21 «Poésie», 922 ; nous soulignons.

22 «Épopée», 512 ; nous soulignons. 
promotion de l'énonciateur, dans le cadre d'une réforme de la poésie épique proposée par Marmontel, est en relation étroite avec cette attention portée au contexte pragmatique. La voix de l'auteur devrait se faire entendre davantage ; «les réflexions et les sentiments du poète font partie des mœurs de l'épopée», comme le chœur fait partie de la structure de la tragédie ancienne ${ }^{23}$. Dans la Pharsale, «[l]e poète [à l'occasion de la représentation d'une rencontre entre les soldats des deux armées ennemies, qui se reconnaissent et s'embrassent] saisit ce moment pour reprocher à ceux de César leur coupable obéissance.

Lâches, pourquoi gémir ? pourquoi verser des larmes?

Qui vous force à porter ces parricides armes ${ }^{24}$ ?»

L'intrusion du discours du poète produit un effet pathétique saisissant, à condition toutefois qu'il donne l'impression d'une parfaite authenticité. C'est l'accord entre l'énoncé et l'énonciation qui fait la valeur unique de ce discours. Marmontel l'explique dans son apologie d'une épopée régénérée :

Mais, dira-t-on, si le rôle du chœur rempli par le poète était une beauté dans l'épopée, pourquoi Lucain serait-il le seul des poètes anciens qui l'aurait fait? Pourquoi ? parce qu'il est le seul que le sujet de son poème ait intéressé vivement. Il était Romain, il voyait encore les traces sanglantes de la guerre civile : ce n'est ni l'art ni la réflexion qui lui a fait prendre le ton dramatique, c'est son âme, c'est la nature même; et le seul moyen de l'imiter dans cette partie, c'est de s'affecter comme lui ${ }^{25}$.

L'inventionéthiquesedoubleicid'une invention formelle :l'intervention du poète dans la narration. Elle se fonde sur l'intensité d'une expérience vécue, sur un caractère "romain", républicain, épris de liberté - non sur un artifice littéraire. Le passage est révélateur cependant de la superposition, fréquente chez Marmontel, d'un langage moderne («ce n'est pas

23 Cette innovation suggérée par la réussite de Lucain va à l'encontre des prescriptions d'Aristote: Homère "est le seul d'entre les poètes à ne pas ignorer ce que doivent être ses interventions personnelles. Le poète doit en effet parler le moins possible en son nom personnel, puisque lorsqu'il le fait, il n'imite pas» (Aristote, Poétique, chap. 24, 1460a 5-10, introduction, traduction et annotation de Michel Magnien, Paris, Le Livre de poche classique, 1990, p. 125).

24 «Épopée», 517 ; nous soulignons.

25 Ibid., 518 ; nous soulignons. 
l'art [...], c'est son âme») et d'un langage rhétorique traditionnel, qui fait intervenir dans ce cas précis la notion de l'ethos de l'orateur, renvoyant de façon ambiguë à un caractère et à une technique. "Le seul moyen de l'imiter, c'est de s'affecter comme lui» : faut-il comprendre que la sincérité s'impose à l'imitateur de Lucain (mais le conseil est inutile, la sincérité excluant toute discipline), ou qu'une émotion artificielle et de commande produit le même "effet de sincérité» qu'une émotion réelle ? Dans ce dernier cas, Marmontel ne ferait que reconduire le précepte rhétorique d'Horace, «si tu veux que je pleure, pleure toi-même». Il semble en réalité que la culture rhétorique autorise la formulation d'une pensée novatrice, qui a provisoirement besoin pour se constituer, et pour se rendre intelligible, du secours de la tradition.

L'invention éthique que Marmontel attribue à Lucain, Fénelon ou Voltaire dans le domaine de l'écriture épique, à Tacite dans le domaine de l'historiographie ${ }^{26}$, implique la défense d'une morale universelle et un ancrage énonciatif dans une situation historique donnée. Elle suppose aussi, à en juger par le "panthéon» des auteurs, plus encore que l'extraterritorialité, une position énonciative de résistant. Lucain écrivant dans la cour de Néron devait «s'attendre à mourir jeune». Marmontel lui-même compose Les Incas pour combattre le fanatisme, après «l'affaire Bélisaire» qui le met aux prises avec les théologiens de la Sorbonne, et pour dénoncer «le roi de guerre», dont la politique permet le massacre des peuples ; son invention ne prend pas la forme du discours abrupt du poète romain, faisant effraction dans la narration, mais celle de situations et de personnages totalement inédits dans le corpus des épopées. Mentionnons quelques-uns de ces personnages: Las Casas, héros pacifiste dressé contre sa communauté, qui refuse de cautionner, par sa présence auprès des Espagnols, la conquête et la destruction du Pérou ; le jeune Espagnol Alonzo de Molina, qui déserte l'armée des conquistadors et combat avec les Indiens contre son propre camp, par fidélité à des valeurs morales universelles ; le conquérant Pizarre, personnage divisé, ambigu, conscient de commettre des crimes et persévé-

26 «'aime donc la manière ingénue et simple de Tacite qui, à chaque trait de burin, nous fait sentir ce qu'il a éprouvé lui-même», quand il dévoile «les profondes noirceurs de l'âme de Tibère, les turpitudes d'Agrippine, la férocité de Néron» («Histoire», 621). "Comme [Tacite] est toujours l'ami ardent de la vertu, l'ami tendre de l'innocence dans le malheur, et l'ennemi austère et inflexible du crime heureux ! Or c'est ce caractère de moralité répandu dans l'Histoire, et surtout dans les Annales de Tacite, qui en fait le prix inestimable» («Histoire», 623). Il est vrai que, dans le cas de Tacite, l'invention ne se détache pas sur fond d'imitation, Marmontel se livrant à des comparaisons entre les historiens romains sans indiquer entre eux de liens de filiation. 
rant néanmoins dans son entreprise. Les personnages d'objecteurs de conscience, sans être incompatibles avec l'univers épique, constituent une invention dans le cadre du système de valeurs qui régit habituellement l'épopée ; le héros contradictoire, dont les actes ne répondent pas à son propre idéal de grandeur morale, est un personnage romanesque et anti-épique. L'imitation du genre de l'épopée, manifeste à travers le choix du sujet (les peuples primitifs américains se substituant aux peuples de l'Antiquité gréco-romaine), la composition du récit (fable «implexe», début in medias res suivi d'un retour en arrière), la mise en scène de phénomènes cosmiques, le recours à l'allégorie ou le style poétique ${ }^{27}$, $\mathrm{s}^{\prime}$ accompagne de l'affirmation d'un engagement éthique de $\mathrm{l}^{\prime}$ auteur ${ }^{28}$ et d'un ensemble de transformations diégétiques, axiologiques, etc., dont nous n'avons pu donner ici qu'un aperçu.

La valorisation d'une position énonciative d'extraterritorialité se vérifie à deux niveaux, celui de la production de la fiction et celui, interne, du scénario fictionnel. Les discours des personnages «porte-parole» de l'auteur, Las Casas et, dans une certaine mesure, Alonzo de Molina, valent principalement par leur «originalité éthique» : originalité en rapport avec une situation de marginalité assumée par rapport à la société, qui autorise un discours critique sur la violence des institutions, et le dévoilement de vérités morales ${ }^{29}$. Il est vrai que dans le cas de l'auteur comme dans celui des personnages, la singularité de la situation d'énonciation est compensée par la portée universelle de l'énoncé ; la littérature moderne réunira, bien plus que ne le font les œuvres du $\mathrm{XVIII}^{\mathrm{e}}$ siècle, position anticonformiste et langage individuel.

Marmontel transpose dans la littérature des pratiques proprement rhétoriques. Les valeurs «moralo-esthétiques» des Éléments rappellent

27 Voir Jean-Marie Roulin, L'épopée de Voltaire à Chateaubriand : poésie, histoire et politique, Oxford, Voltaire Foundation, coll. "Studies on Voltaire and the Eighteenth Century », 2005 (p. 101-124, consacrées à Marmontel); et "Les Incas de Marmontel, ou comment être un poète philosophe», dans Pierre Frantz (dir.), L'épique : fins et confins, Besançon, Presses Universitaires Franc-Comtoises, 2000, 193-206.

28 «Le but de cet ouvrage est donc, je l'annonce sans détour, de contribuer, si je le puis, à faire détester de plus en plus ce fanatisme destructeur ; d'empêcher, autant qu'il est en moi, qu'on ne le confonde jamais avec une religion compatissante et charitable» (Les Incas, éd. cit., « Préface », t. 1, 17).

29 Il en est de même dans Bélisaire, qui n'est pas une imitation d'un modèle littéraire antique, mais qui conforte l'idée d'une prédilection de Marmontel pour des positions énonciatives atypiques : le héros de ce roman est un proscrit, victime du pouvoir politique. 
le docere de la rhétorique, et la traditionnelle utilité attribuée à l'œuvre ${ }^{30}$. L'écrivain, comme l'orateur, vaut par sa fidélité au moment présent, par ce qu'il laisse transparaître de son humanité individuelle, par la puissance émotive de son discours.

La «rhétoricisation» de la littérature apparaît comme une voie de modernisation. Sous couvert d'un langage ancien, les Éléments travaillent à assouplir - jusqu'à le subvertir - l'idéal classique de «beauté universelle» et de perfection esthétique, qui implique une dévaluation de la singularité énonciative. La rhétorique invoquée par Marmontel est à certains égards une rhétorique reconstruite, redéfinie, adaptée aux besoins de temps nouveaux ${ }^{31}$. Elle est purifiée, notamment, de sa propension à la manipulation et de sa connivence avec le mensonge : l'article «Orateur» s'emploie à promouvoir une éloquence qui soit le témoignage de l'âme. Le discours de l'orateur idéal est la manifestation transparente d'un caractère véritable et d'une expérience vécue ${ }^{32}$. «L'originalité éthique» moderne, qui prend toute sa signification dans un contexte monarchique, ne se confond pas avec la vertu de l'antique orateur républicain. La rhétorique des Éléments sert en définitive à ouvrir l'espace du classicisme, et elle-même connaît des transformations qui l'intègrent dans un système de valeurs moderne.

30 Sur les rapports que Marmontel entretient avec la rhétorique, voir Jean-Paul Sermain, «Marmontel et la réforme de la rhétorique», dans Kees Meerhoff et Annie Jourdan (dir.), Mémorable Marmontel. 1799-1999, Amsterdam et Atlanta, Rodopi, coll. «Cahiers de recherches des instituts néerlandais de langue et de littérature françaises», 1999, 165-174. J.-P. Sermain montre que Marmontel maintient vivante la rhétorique antique (non seulement l'elocutio, mais les principes fondamentaux de l'inventio et de la dispositio) et qu'il tente de l'adapter à la situation contemporaine, par le recensement des types d'éloquence qui restent praticables à l'époque moderne (l'éloquence du barreau, de la chaire, et surtout le genre de l'éloge académique, décrit comme une nouvelle forme d'éloquence populaire).

31 Voir Marc-André Bernier, «Marmontel, lecteur de Cicéron : histoire et invention poétique dans les Éléments de littérature (1787)», dans The Electronic Journal of the Canadian Society for Aesthetics / La revue électronique de la Société canadienne d'esthétique, vol.12, été 2006, http://www.uqtr.uqutr.uquebec.ca/AE/Vol_12/ Dumouchel/bernier.htm.

32 Notre interprétation s'écarte ici de celle de Jean-Paul Sermain, qui considère cette «épuration" morale de la rhétorique comme une concession aux goûts dominants de l'époque, dans le cadre d'une stratégie de défense de la rhétorique («Marmontel et la réforme de la rhétorique», art. cit., p. 170). La tendance «à cantonner l'éloquence au domaine du cœur» s'accorde avec la tonalité dominante de l'œuvre de Marmontel tout entière ; $l$ 'horreur de la sophistique est véritablement un credo de l'auteur. 
La promotion de la nouveauté et de « l'expérimentation " générique, annonçant les audaces de la littérature romantique, était promise à un brillant avenir. En revanche, l'exaltation de la sincérité, du courage, de l'engagement - valeurs qui coexistent dans les Éléments avec l'idéal de perfection - appartient en propre à la dernière génération des Lumières, celle de Louis-Sébastien Mercier, de Jean-Louis Carra et de bien d'autres. L'invention moderne par excellence dans les années 1760-1780 réside dans la fidélité de ces «orateurs muets, indépendants, intrépides» que sont les écrivains ${ }^{33}$ à une vocation politique et morale. C'est à la fois la noblesse et la fragilité de cette invention particulière que de dépendre si étroitement de la situation historique dans laquelle elle s'inscrit. Pour que se produise le miracle de la communication littéraire, il faut un public contemporain de l'auteur - d'une contemporanéité réelle ou «spirituelle», «par analogie», en quelque sorte. Alors, l'écrivain est «le grand homme» dont l'œuvre éveille dans l'âme du lecteur des sentiments de fraternité et de reconnaissance.

YOUMNA CHARARA

Paris

33 L'expression est de Mercier, L'An 2440. Rêve s'il en fut jamais (1770), éd. A. Pons, Paris, Adel, «Bibliothèque des Utopies», 1977, ch. 29, «Les gens de lettres», p. 173 ; voir aussi le ch. 28, «La bibliothèque du roi». 